\title{
Review \\ Monitoring trauma and intensive care unit resuscitation with tissue hemoglobin oxygen saturation
}

\author{
Rachel J Santora ${ }^{1}$ and Frederick A Moore ${ }^{1,2}$
}

\author{
1Department of Surgery, The Methodist Hospital, 6550 Fannin Street, SM 1661, Houston, TX 77030, USA \\ 2Department of Surgery, Weill Cornell Medical College, 525 E. 68th Street, New York, NY 10065, USA
}

Corresponding author: Rachel J Santora, rjsantora@tmhs.org

Published: 30 November 2009

This article is online at http://ccforum.com/content/13/S5/S10

Critical Care 2009, 13(Suppl 5):S10 (doi:10.1186/cc8008)

(c) 2009 BioMed Central Ltd

\begin{abstract}
Introduction: The purpose of the present review is to review our experience with near-infrared spectroscopy (NIRS) monitoring in shock resuscitation and predicting clinical outcomes.

Methods: The management of critically ill patients with goaloriented intensive care unit (ICU) resuscitation continues to evolve as our understanding of the appropriate physiologic targets improves. It is now recognized that resuscitation to achieve supranormal indices is not beneficial in all patients and may precipitate abdominal compartment syndrome.

Results: Over the years, ICU technology has provided physicians with specific physiologic parameters to guide shock resuscitation. Throughout this time, the tissue hemoglobin oxygen saturation $\left(\mathrm{StO}_{2}\right)$ monitor has emerged as a non-invasive means to obtain reliable physiologic parameters to guide clinicians' resuscitative efforts. $\mathrm{StO}_{2}$ monitors have been shown to aid in early identification of nonresponders and to predict outcomes in hemorrhagic shock and ICU resuscitation. These data have also been used to better understand and refine existing resuscitation protocols. More recently, use of NIRS technology to guide resuscitation in septic shock has been shown to predict outcomes in high-risk patients.

Conclusions: $\mathrm{StO}_{2}$ is an important tool in identifying high-risk patients in septic and hemorrhagic shock. It is a non-invasive means of obtaining vital information regarding outcome and adequacy of resuscitation.
\end{abstract}

\section{Introduction}

Near-infrared spectroscopy (NIRS) offers a new monitoring tool to be used in critically ill patients. NIRS measures the saturation of hemoglobin in the skeletal muscle and serves as an index of perfusion. We have studied the value of tissue hemoglobin oxygen saturation $\left(\mathrm{StO}_{2}\right)$ in a number of settings, including intensive care unit (ICU) shock resuscitation, predicting outcomes in the emergency department (ED), and to better understand massive transfusion (MT). The present article will briefly review these experiences.

\section{Intensive care unit traumatic shock resuscitation}

In the 1980s William Shoemaker and colleagues wrote a series of papers addressing the use of physiologic monitoring to predict outcome and to assist in clinical decision-making [1-3]. Two interesting variables identified were oxygen delivery $\left(\mathrm{DO}_{2}\right)$ and oxygen consumption. In one observational study, Shoemaker and colleagues tracked these variables in preoperative and postoperative periods of high-risk patients undergoing elective surgical procedures. In the preoperative period, normal $\mathrm{DO}_{2}$ is $450 \mathrm{ml} / \mathrm{min} / \mathrm{m}^{2}$ [1]. Following operative intervention, survivors will increase their $\mathrm{DO}_{2}$ to above $600 \mathrm{ml} / \mathrm{min} / \mathrm{m}^{2}$ [1]. Nonsurvivors attempt to achieve this similar hyperdynamic state but cannot sustain it. Similarly, normal oxygen consumption is approximately $120 \mathrm{ml} / \mathrm{min} / \mathrm{m}^{2}$; following an operation, survivors increase their oxygen consumption to supernormal levels of above $150 \mathrm{ml} / \mathrm{min} / \mathrm{m}^{2}$. Likewise, nonsurvivors attempt to increase their oxygen consumption in this range but are not able to achieve it. Based on these observations, Shoemaker and colleagues concluded that survivor parameters include cardiac index $>4.5 \mathrm{l} / \mathrm{min} / \mathrm{m}^{2}, \mathrm{DO}_{2}>600 \mathrm{ml} / \mathrm{min} / \mathrm{m}^{2}$, and oxygen consumption $>150 \mathrm{ml} / \mathrm{min} / \mathrm{m}^{2}$ [1]. They proposed that the survivor parameters should be adopted as resuscitation goals and popularized the term supranormal oxygen delivery.

In the late 1980s Shoemaker and colleagues published the results of a prospective trial where there were three study groups who were managed by three different strategies [2]. The first group received a central venous line, and central venous pressures were used at the discretion of clinicians to

$\mathrm{ACS}=$ abdominal compartment syndrome; $\mathrm{CT}=$ computerized tomography; $\mathrm{DO}_{2}=$ oxygen delivery; $\mathrm{ED}=$ emergency department; $\mathrm{FFP}=$ fresh frozen plasma; ICU = intensive care unit; ISS = injury severity score; MOF = multiple organ failure; MT = massive transfusion; NIRS = near-infrared spectroscopy; $\mathrm{PA}=$ pulmonary artery $\mathrm{StO}_{2}=$ tissue hemoglobin oxygen saturation; $\mathrm{SvO}_{2}=$ mixed venous hemoglobin oxygen saturation. 
guide resuscitation. Pulmonary artery (PA) catheters were placed in the second group, and monitored variables (PA wedge pressure and cardiac index) were again used at the clinicians' discretion to direct resuscitation. PA catheters were also placed in the third group of patients; however, these patients were managed with a resuscitative protocol that emphasized early volume loading followed by inotropic support with dobutamine. The results of this trial showed a dramatic reduction in mortality from 23\% and 35\% down to $4 \%$ between the central venous pressure and PA control groups and the PA protocol-treated group, respectively [2].

Based on these observations and others, Shoemaker and colleagues proposed that unrecognized flow-dependent oxygen consumption contributed to the development of multiple organ failure (MOF) [2,3]. At this time, MOF was the leading cause of late ICU deaths and its pathophysiology was unclear. This theory attributed myocardial dysfunction that occurs in patients in shock as an important cause of subsequent MOF. This concept that unrecognized flow-dependent oxygen consumption could be corrected by maximizing $\mathrm{DO}_{2}$ became a popular strategy.

Meanwhile, new technology was being introduced into the ICU - including continuous venous oximetry and continuous cardiac output monitoring with PA catheters [4]. This permitted widespread use of oxygen transport variables to guide resuscitation. At the Denver General Hospital in the early 1990s, surgical intensivists developed a bedside clinical protocol that involved identification of patients who were at risk for postinjury MOF [5]. Upon arrival in the ICU, a PA catheter was presumptively placed in high-risk patients and a series of escalating interventions was utilized to maximize the patient's $\mathrm{DO}_{2}$. Based on the 12-hour response to these interventions, the intensivists could predict who would develop MOF.

A number of prospective randomized trials were performed in the 1990s to test whether supernormal resuscitation truly reduced mortality in critically ill patients [6]. The results of these studies were varied and suggested that there are subgroups of patients who do benefit from this strategy. Benefits of hemodynamic optimization were most readily observed in acutely ill patients who had not succumbed to end-organ failure.

In the late 1990s at the University of Texas Houston Medical School, a team of surgical intensivists collaborated with bioengineers and health information experts to further refine the logic for traumatic shock resuscitation and implemented it with a computerized clinical decision support application [7]. Figure 1 represents an overview of this protocol. Patients meeting specific criteria (evidence of major torso trauma, evidence of shock as documented by base deficit $>6 \mathrm{mEq}$, and anticipated blood transfusions $>6$ units in 12 hours) had PA and peripheral artery catheters inserted upon ICU arrival.
They were resuscitated to a $\mathrm{DO}_{2}$ goal of $600 \mathrm{ml} / \mathrm{min} / \mathrm{m}^{2}$ with a series of escalating interventions to achieve this goal in nonresponders. This became the standard of care in the shock trauma ICU at the Memorial Hermann Hospital in Houston, Texas. The protocol provided a unique opportunity to prospectively collect data on how patients responded to interventions and to further refine the existing protocol to optimize resuscitation.

This protocol also provided the opportunity to test the utility of various monitors in this process of care. One such monitor was $\mathrm{StO}_{2}$. To evaluate skeletal and subcutaneous $\mathrm{StO}_{2}$ changes as surrogates for $\mathrm{DO}_{2}$ l changes and to compare these variables with other commonly used indices of shock resuscitation, we conducted a prospective study using $\mathrm{StO}_{2}$ monitors in shock resuscitation [8]. Figure $2 \mathrm{a}, \mathrm{b}$ represents the variables tracked over the first 24 hours of ICU admission. These included $\mathrm{StO}_{2}$ at $6 \mathrm{~mm}$ (subcutaneous), $\mathrm{StO}_{2}$ at $20 \mathrm{~mm}$ (skeletal muscle), mixed venous hemoglobin oxygen saturation $\left(\mathrm{SvO}_{2}\right)$ (derived from the PA catheter), and serial lactate levels and base deficit levels (both obtained from serial blood testing). These variables were monitored simultaneously in severely injured patients undergoing protocol-directed shock resuscitation. Upon arrival in the ICU, $\mathrm{DO}_{2}$ averaged approximately $400 \mathrm{ml} / \mathrm{min} / \mathrm{m}^{2}$ and was driven by the protocol intensivists to a maximum of roughly $700 \mathrm{ml} / \mathrm{min} / \mathrm{m}^{2}$ by 24 hours. Throughout this resuscitation, skeletal muscle $\mathrm{StO}_{2}$ (at $20 \mathrm{~mm}$ ) appears to be quite responsive to changes in systemic $\mathrm{DO}_{2}$. Changes in $\mathrm{DO}_{2}$ resulted in a predictable decrease in lactate and base deficit levels, signifying effective shock resuscitation. Subcutaneous $\mathrm{StO}_{2}$ (at $6 \mathrm{~mm}$ ) was generally unresponsive to the resuscitation intervention, while $\mathrm{SvO}_{2}$ derived from the PA catheter shows only a small rise from roughly 70 to $78 \%$ during the resuscitation process. Changes in $\mathrm{StO}_{2}(20 \mathrm{~mm})$ showed a strong correlation with changes in $\mathrm{DO}_{2}$, base deficit, and lactate ( $r=0.95$ vs. 0.83 vs. 0.82 , respectively) but only modest correlation with $\mathrm{SvO}_{2}(r=0.55)$.

The observation that the $\mathrm{StO}_{2}$ is an index of profusion that tracks $\mathrm{DO}_{2}$ during active resuscitation led laboratory investigators to investigate the role of $\mathrm{StO}_{2}$ monitors in the setting of hemorrhagic shock. Greg Beilman and colleagues at the University of Minnesota looked at the use of $\mathrm{StO}_{2}$ as an early determinate of irreversible shock [9]. Monitored pigs were subjected to a hemorrhagic shock protocol involving removal of $35 \%$ of the pig's blood volume over a 90-minute period. Resuscitation was done in a stepwise fashion, with administration of a $20 \mathrm{ml} / \mathrm{kg}$ bolus of lactated Ringer's solution every 30 minutes, for a total of four boluses.

Eighteen out of 20 animals survived 90 minutes of hemorrhagic shock: 12 of the animals survived resuscitation (resuscitatable) and six animals developed irreversible shock (nonresuscitatable). Hemodynamic and NIRS measurements were compared between the groups (resuscitatable vs. 


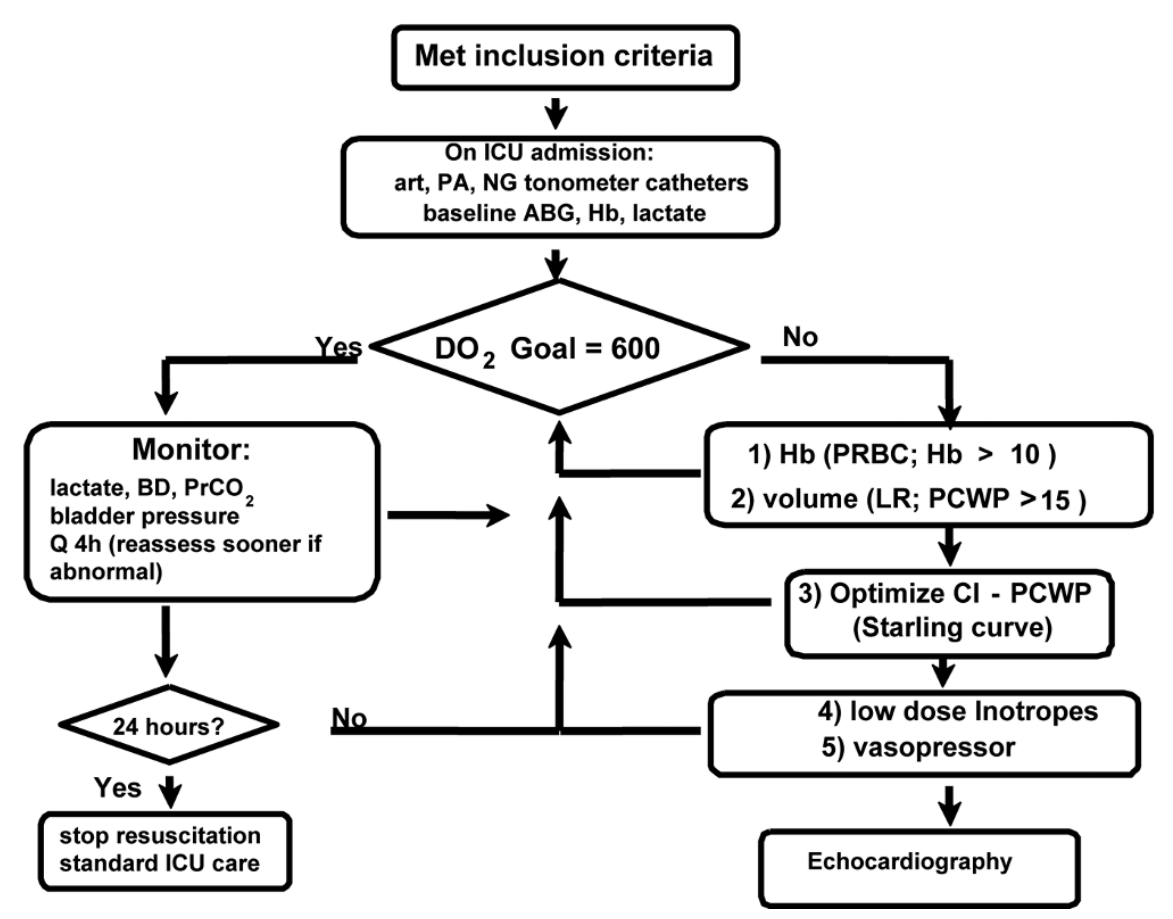

Overview of the resuscitation protocol. $\mathrm{ABG}$, arterial blood gas; art, arterial; $\mathrm{BD}$, base deficit; $\mathrm{Cl}$, cardiac index; $\mathrm{DO}_{2}$, oxygen delivery; $\mathrm{Hb}$, hemoglobin; ICU, intensive care unit; LR, lactated Ringer's solution; NG, nasogastric; PA, pulmonary artery; PCWP, pulmonary wedge pressure; PRBC, packed red blood cells; $\mathrm{PrCO}_{2}$, regional carbon dioxide tension measured by gastric tonometry. Reproduced with permission from [7].

nonresuscitatable) at each step of resuscitation. All animals had a decrease in cardiac output, $\mathrm{SvO}_{2}$ and $\mathrm{DO}_{2}$ and an increase in lactate. A decrease in skeletal muscle, liver and stomach $\mathrm{StO}_{2}$ was also observed. Beyond 30 minutes of resuscitation, reversible shock patients were seen to have a steady increase in cardiac output, while irreversible shock patients demonstrated a steady decrease. More notably, the drop in skeletal $\mathrm{StO}_{2}$ was significantly greater in animals who did not survive resuscitation. Measurements of hind limb $\mathrm{StO}_{2}$ in each group diverged within 30 minutes of shock, such that by the end of the 90-minute period the $\mathrm{StO}_{2}$ value for the nonresuscitatable group remained low despite resuscitation. Animals destined to survive shock and resuscitation did not exhibit an irreversible decline in $\mathrm{StO}_{2}$. These findings demonstrate skeletal $\mathrm{StO}_{2}$ as a reliable, non-invasive means for early differentiation between resuscitatable and nonresuscitatable animals.

Another investigative group from the University of Miami, led by Stephen Cohn, was also interested in the use of noninvasive $\mathrm{StO}_{2}$ to guide fluid resuscitation after traumatic shock. They evaluated three resuscitative strategies in a series of three in vivo hemorrhagic shock models with or without a simultaneous penetrating femur injury [10]. After a 30-minute shock period, animals were randomized to receive no resuscitation, to receive $15 \mathrm{ml} / \mathrm{kg}$ Hextend or to receive shed blood $+20 \mathrm{ml} / \mathrm{kg}$ lactated Ringer's solution. Serial lactate levels and serial base deficit levels were used to identify the best response to resuscitation. Of the three interventions, Hextend had the best clearance of base deficit and lactate levels over the ensuing 150 minutes of observation. $\mathrm{SvO}_{2}$ (derived from an invasive PA catheter) also identified colloid resuscitation with Hextend as an effective resuscitative strategy within 30 minutes of resuscitation. Similarly, hind limb $\mathrm{StO}_{2}$ (a non-invasive monitor) indicated enhanced resuscitation in response to Hextend.

Taken together, these clinical and research data suggest that $\mathrm{StO}_{2}$ (derived from a non-invasive monitor) could provide information about the effectiveness of resuscitation equivalent to that of an invasive PA catheter or serial blood draws to measure base deficit or lactate levels.

\section{Predicting outcomes}

In the late 1980s, Jim Davis and his colleagues from San Diego popularized early routine collection of the base deficit in trauma patients to evaluate for possible shock and to guide ensuing volume resuscitation [11]. In subsequent publications they demonstrated that the admission base deficit in trauma patient predicts transfusion requirements, risk of complications and mortality [12]. Interestingly, Sauaia and colleagues - who were interested in identifying early 

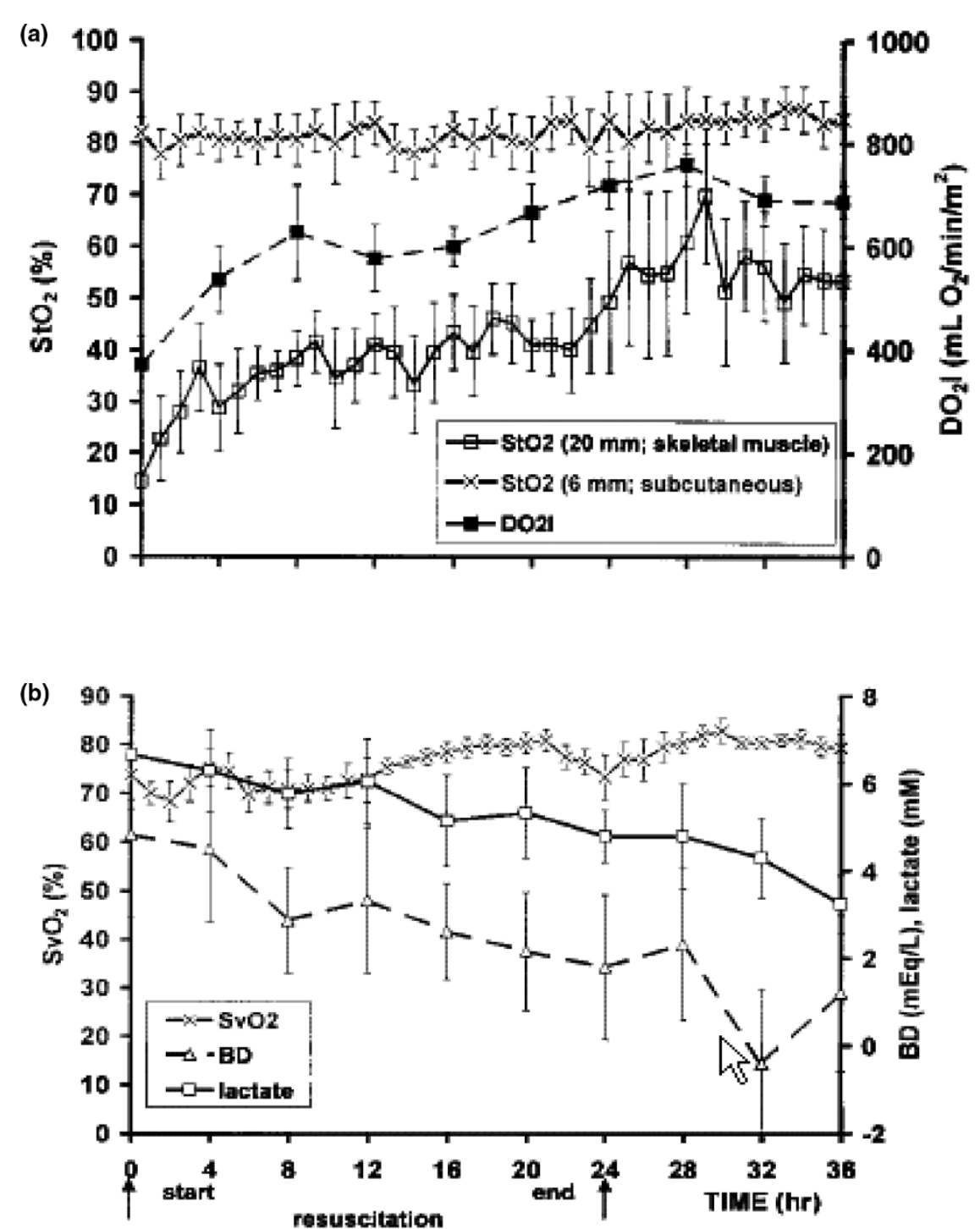

\footnotetext{
Shock resuscitation variables during shock resuscitation (first 24 hours) and the following 12 hours. (a) Tissue hemoglobin oxygen saturation $\left(\mathrm{StO}_{2}\right)$, deltoid skeletal muscle and subcutaneous $\mathrm{StO}_{2}$ saturation, monitored non-invasively using a prototype near-infrared spectrometer (Biospectrometer-NB Oximeter; Hutchinson Technology, Inc., Hutchinson, MN, USA). Do 2 l, systemic oxygen delivery index. (b) Mixed venous hemoglobin oxygen saturation $\left(\mathrm{SvO}_{2}\right)$ monitored invasively using a pulmonary artery catheter with fiberoptic oximetry capability. BD, base deficit; lactate, serum lactate concentration. Reproduced with permission from [8].
}

predictors of postinjury MOF - identified the ED base deficit as the earliest independent predictor of MOF [13]. This observation was validated in a second study from the same group published in the late 1990s [14]. These data indicated that the initial shock insult in a trauma patient is an important determinate of adverse outcomes, particularly the development of MOF.

With this in mind, Cohn and colleagues decided to perform a study using $\mathrm{StO}_{2}$ monitoring in the emergency room to determine whether it could predict MOF [15]. They performed a prospective observational study involving seven
US trauma centers over a 16-month time period, ending in 2006. They proposed that thenar $\mathrm{StO}_{2}$ and base deficit could equally predict MOF and death in major torso trauma patients presenting in shock. Entry criteria included major torso trauma (excluding severe head injury), ED shock (systolic blood pressure $<90 \mathrm{mmHg}$ or base deficit $\geq 6 \mathrm{mEq} / \mathrm{l}$ ), and requirement of a blood transfusion. The $\mathrm{StO}_{2}$ monitor was placed within 30 minutes of arrival in patients meeting the inclusion criteria. Data collection included demographics (age, gender, mechanism injury, injury severity score (ISS)), continuous $\mathrm{StO}_{2}$ monitoring for the first 24 hours and routine shock and resuscitation parameters. They looked at MOF and 
death as their primary outcomes. There were 381 study patients enrolled, predominantly males, with a high ISS of $28 \pm 15$. Two-thirds of patients sustained blunt mechanism injury, most arrived in severe shock as documented by admission systolic blood pressure of $84 \pm 22 \mathrm{mmHg}$, heart rate of $120 \pm 23$ beats/minute and $E D$ base deficit of $9 \pm 5 \mathrm{mEq} / \mathrm{l}$, and they received on average $8 \pm 7$ units of packed red cells within the first 6 hours.

Figure 3 a depicts the receiver operator curve for the ability of $\mathrm{StO}_{2}$ base deficit and systolic blood pressure to predict the MOF outcome. MOF occurred in 50 out of the 381 cases and it appeared that $\mathrm{StO}_{2}$ performed equally as well as base deficit and systolic blood pressure, with an area under the curve of $0.66,0.63$ and 0.57 , respectively. Figure $3 b$ depicts the receiver operator curve for the endpoint of death. There were 55 deaths out of 381 study patients, and it appeared that $\mathrm{StO}_{2}$ outperformed base deficit and systolic blood pressure in predicting this outcome with an area under the curve of 0.72 versus 0.67 versus 0.66 , respectively [15].

When these data were presented at the 2006 annual meeting of the American Association for the Surgery for Trauma, one question asked was whether $\mathrm{StO}_{2}$ measurements compared with lactate levels as a predictor of MOF and mortality. With this question in mind, the study database was re-analyzed to identify patients who had lactate and $\mathrm{StO}_{2}$ measured in the first hour [16]. In this subgroup analysis, 151 patients had a documented lactate level. Twenty-seven of these patients developed MOF. The corresponding $\mathrm{StO}_{2}$ levels had a predictive power that was equivalent to lactate levels, with an area under the curve of 0.64 versus 0.65 , respectively. Looking at death as a primary endpoint, 26 deaths occurred in this cohort and again $\mathrm{StO}_{2}$ as a predictor outperformed lactate in predicting death, with an area under the curve of 0.77 versus 0.71 [16].

From these data we conclude that $\mathrm{StO}_{2}$ obtained within the first hour after ED admission is an equally reliable predictor of adverse outcomes when compared with the more conventionally used parameters of lactate and base deficit. $\mathrm{StO}_{2}$, however, is obtained non-invasively and continuously. This allows the clinician to quickly identify patients in shock, who are at high risk for adverse outcomes and to assess adequacy and response to resuscitation.

\section{Improving the understanding of epidemiology of massive transfusion}

In the 1980s US trauma surgeons witnessed tremendous advances in trauma care, including trauma system development, advanced trauma life support, damage control resuscitation and goal-orientated ICU resuscitation. With these advances in trauma care, there was a reduction in patients who bled to death on the operating room table. The cohort of patients with severe bleeding who survived long enough to be admitted into an ICU, however, were at high risk of developing abdominal compartment syndrome (ACS), which emerged in epidemic proportions in the mid 1990s.

To better understand this new syndrome, Zsolt Balogh (a visiting research fellow and Hungarian trauma surgeon) analyzed the prospective shock resuscitation database that was maintained by the University of Texas at Houston trauma research center. He performed a series of four database analyses that tremendously improved our understanding of the pathophysiology of ACS and its relationship to MOF [17-21]. Using prediction models, Balogh could accurately predict who was going to develop ACS within 3 hours of arriving in the ED. He showed that conventional use of highvolume isotonic crystalloids to normalize blood pressure in the ED were harmful to this subgroup of patients, leading to hemodilution and promoting further bleeding. Once these high-risk patients arrive in the ICU, they have significant intraabdominal hypertension - with mean urinary bladder pressures $\geq 19 \mathrm{mmHg}$.

A popular belief at the time was that patients with high intraabdominal pressure (which falsely elevates central venous pressure) and low cardiac output should be volume loaded to increase preload and thereby optimize cardiac output. Balogh's studies, however, nicely demonstrated this is exactly the wrong thing to do in patients with impending ACS. These patients have low cardiac output and high systemic vascular resistance. Additional increases in pre-load with isotonic crystalloid boluses do not increase cardiac output; instead, vigorous volume loading pushes them into full-blown ACS. Balogh also demonstrated that reducing the $\mathrm{DO}_{2}$ goal of the ICU resuscitation protocol from a supranormal level of $600 \mathrm{ml} / \mathrm{min} / \mathrm{m}^{2}$ to a more normal level of $500 \mathrm{ml} / \mathrm{min} / \mathrm{m}^{2}$ was associated with a significant reduction in the incidence of ACS, MOF and death. From these data it was concluded that ACS was the result of early (in the ED) administration of inordinate amounts of crystalloids and that fundamental changes in the early care of patients with severe hemorrhage were necessary.

Trauma surgeons at the University of Texas at Houston and the Memorial Hermann Hospital then focused their efforts on developing new pre-ICU care protocols, including ED resuscitation, a MT protocol, whole-body CT scanning, a pelvic fracture protocol, and the Focused Assessment with Sonography for Trauma (FAST) examination with backup peritoneal aspirate. Out of these protocols, the most significant impact on patient outcome was presented with the MT protocol. Gonzalez and colleagues performed a retrospective analysis of the shock resuscitation database to evaluate the existing MT protocol. MT was defined as $>10$ units of packed red blood cells in 24 hours [22]. This analysis identified that patients arrived in the ICU with irreversible coagulopathy despite adherence to the existing MT protocol. This coagulopathy persisted as the patient went on to require substantial blood transfusion. The admission 

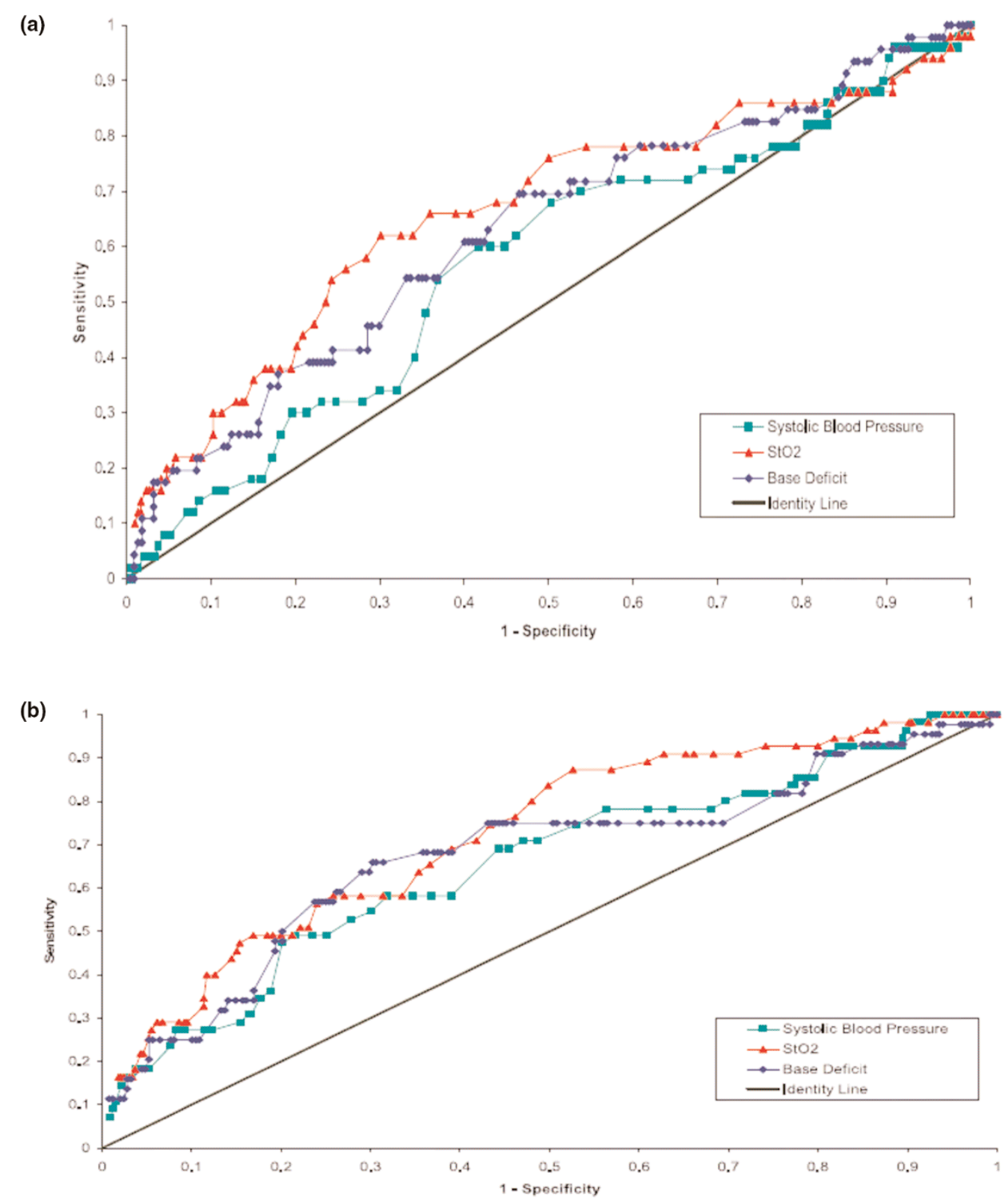

Physiologic parameters relative to multiple organ dysfunction syndrome and mortality. Receiver operating characteristic curves for physiologic parameters relative to (a) multiple organ dysfunction syndrome and (b) mortality within 1 hour of emergency department arrival. Skeletal muscle tissue hemoglobin saturation $\left(\mathrm{StO}_{2}\right)$ monitored non-invasively using a prototype near-infrared spectrometer (InSpectra Tissue Spectrometer; Hutchinson Technology, Inc., Hutchinson, MN, USA). BD, base deficit; SBP, systolic blood pressure. Reproduced with permission from [15].

International Normalized Ratio was highly correlated with subsequent mortality, and the investigators concluded that fresh frozen plasma should be used earlier and more aggressively in the ED for patients that required a MT. They proposed that fresh frozen plasma (FFP) and packed red blood cells be used at a ratio of 1:1.

John Holcomb, who developed the original MT protocol at Memorial Hermann Hospital, was also an advocate for the use of activated factor VII in damage-control patients [23], and subsequently further expanded this concept when he became the Commander of the US Army Institute of Surgical Research in San Antonio. He and his colleagues developed a new concept of damage control resuscitation, which emphasizes direct treatment of coagulopathy in trauma during initial resuscitative efforts [24]. The primary goals of this strategy were early use of FFP, activated recombinant factor VII and fresh whole blood when available and to pursue hypotensive 
resuscitation until hemorrhage is definitively controlled. This concept has been used in the current conflicts in Afghanistan and Iraq. The data emerging from those conflicts suggest that this approach reduces mortality in patients sustaining serious torso and limb trauma.

While these strategies are all very enticing, a central question emerged when faced with the clinical quandary of a patient with an unstable pelvic fracture who is in obvious shock: how much time does the clinician have to make these critical decisions? When do you initiate aggressive FFP administration or administer factor VII? After initial resuscitation, does the clinician triage the patient to the CT scanner, to the operating room or wait for the angiographers to come in to embolize potential pelvic bleeder? To answer these questions, a retrospective analysis of the $\mathrm{StO}_{2}$ database was conducted [25]. The specific aims of the analysis were: to define the current epidemiology of MT, which includes documenting early temporal events and confirming the association of MT with bad outcomes, including MOF and death; and, secondly, to determine feasibility of the early prediction of $\mathrm{MT}$ and the potential role of $\mathrm{StO}_{2}$ in these prediction models.

In this 16-month observational study, there were 381 patients who met entry criteria; 114 (30\%) received MT (defined as $>10$ units in the first 24 hours). The MT cohort versus the non-MT cohort had similar demographics, but the patients who received a MT had a notably higher ISS (32 \pm 17 vs. $26 \pm 15)$. Analysis of the data also showed that the MT patients arrived hypothermic, were in severe shock and were notably coagulopathic. Their initial International Normalized Ratio was $1.7 \pm 1.4$. Comparing baseline characteristics between the two cohorts, all variables with the exception of temperature were significantly less deranged in the non-MT cohort. These data demonstrated that, upon arrival, MT patients are different from non-MT patients. They have higher ISS, they have more severe shock and they are severely coagulopathic prior to aggressive inhospital resuscitation. Most notably, MT is a very rapid process of care; $40 \%$ of the patients met the threshold of 10 units within 2 hours and $80 \%$ met the threshold within 6 hours. By 6 hours the MT cohort had received on average 20 units of packed red blood cells. Looking at time to death in the first 24 hours, there were 26 early deaths in the MT cohort, of which two-thirds occurred within the first 6 hours. These data emphasize that critical decisions must be made in a limited amount of time.

Assessment of clinical outcomes such as ICU-free days, ventfree days, hospital days, and percentages of death and MOF showed that MT patients have longer ICU stays and spend more time on mechanical ventilators. The mortality rate for MT patients was $33 \%$; the incidence of MOF was $31 \%$, and $50 \%$ of patients had the combined outcome of MOF and death. When you compare these outcomes with the non-MT patients, it is clear that MT patients have much worse outcomes. We were also interested in identifying the early predictors of bad outcome (MOF/death). Using univariate analysis comparing good outcome versus bad outcome, a number of variables were identified. We then chose $P<0.15$ between the two groups to identify variables to be placed into three multivariate models using data available 1 hour, 2 hours and 3 hours after their trauma center arrival [26]. The purpose of this analysis was to identify a specific variable that could help the clinicians make critical decisions. More specifically, for patients in whom it is decided to initiate a MT, to determine which variables would tell you that a specific patient was at high risk of dying and therefore would warrant the use of a potentially harmful intervention such as activated factor VIla. In the 1-hour, 2-hour and 3-hour models two variables fell out: injury severity score $>25$ at 1 hour and 2 hours, and the $\mathrm{StO}_{2}$ value in the 1-hour, 2-hour and 3-hour models. $\mathrm{StO}_{2}$ is therefore a good variable to help identify the patient who is going to die.

Given the amount of controversy that currently exists over the management of MT and the need for a clinical trial to address the potential role of aggressive early FFP analysis, we performed a second follow-up analysis of the $\mathrm{StO}_{2}$ database with a focused study on the "MT died early" cohort [26]. We looked at the 114 patients who received a MT and divided them into three groups: G1 was the 27 early deaths, G2 included 31 patients who developed MOF or died late, and G3 was the remaining 50 patients who had a MT but did not die.

Looking at the number of packed red blood cells over the first 6 hours for the various groups, the G1 MT died early group received 26.4 units of packed red blood cells but only 6.5 units of FFP. Considering the bloody vicious cycle pathophysiology and comparing the three groups for temperature, acidosis and coagulopathy, the G1 group had more deranged physiology. This group was severely acidotic and coagulopathic in comparison with G2 and G3. Interestingly, the coagulopathy in G1 was noted to worsen over the first 3 hours; the MT died early patients arrived with a significantly higher International Normalized Ratio of 2.4, which increased to 3.8. Consequently, approximately $70 \%$ of these patients bled to death during a time period corresponding to their worsening coagulopathy [26]. From these data we conclude that this subgroup of patients arrived with coagulopathy, received an ordinary amount of packed red blood cells (that is, 26.4 units) but received only 6.5 units of FFP, and therefore their coagulopathy was not treated.

When we look at the shock parameters (Figure 4) of systolic blood pressure, base deficit and $\mathrm{StO}_{2}$, the major observation is that $\mathrm{StO}_{2}$ is very different in this cohort of patients who were dying early. $\mathrm{StO}_{2}$ measurements dropped steadily from 70 to $50 \%$ as they died early from exsanguination. We therefore concluded that, in the setting of MT, a dropping $\mathrm{StO}_{2}$ portends early death from exsanguination. 

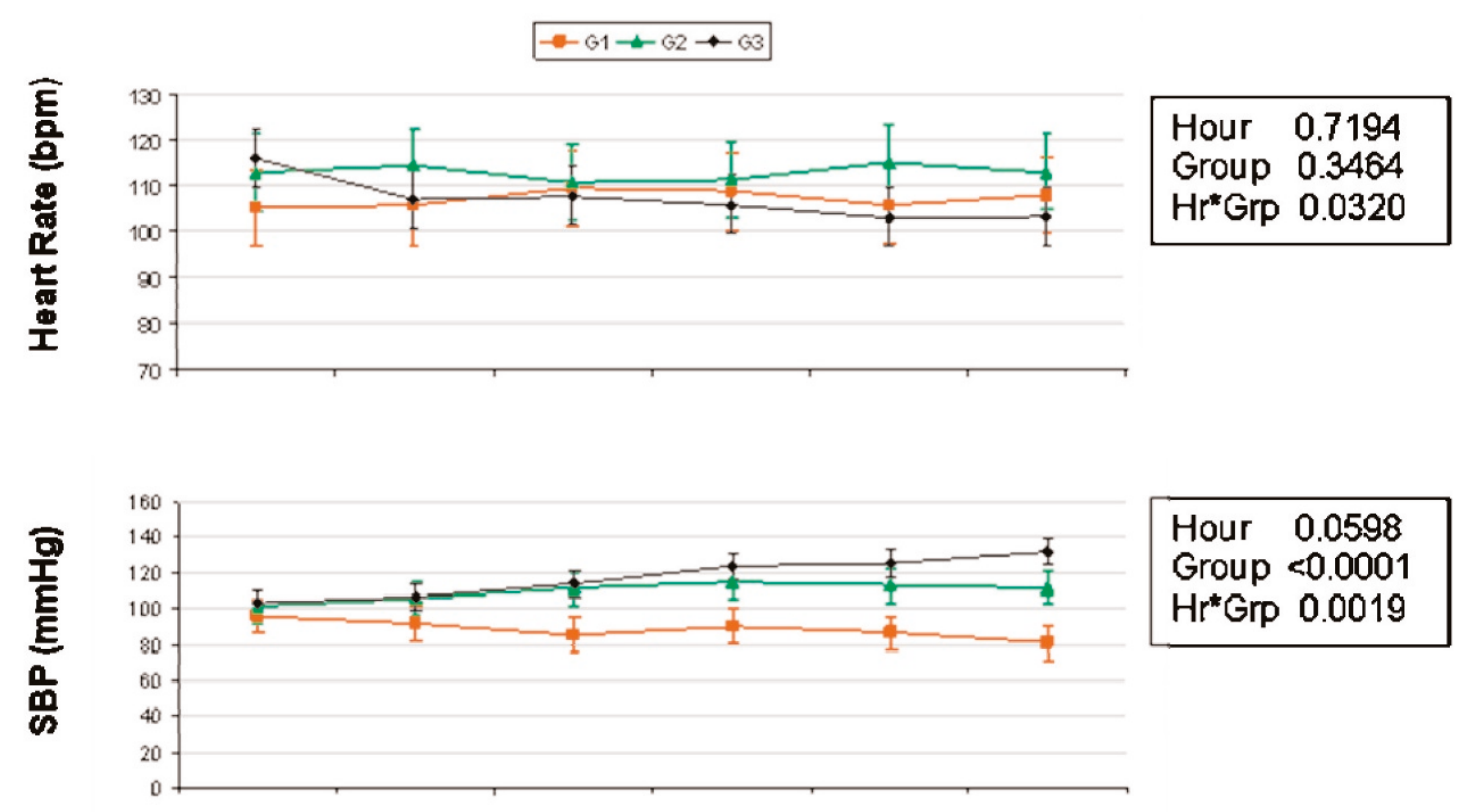

\section{Hour $\quad 0.0598$ Group $<0.0001$ $\mathrm{Hr}{ }^{\star}$ Grp 0.0019}

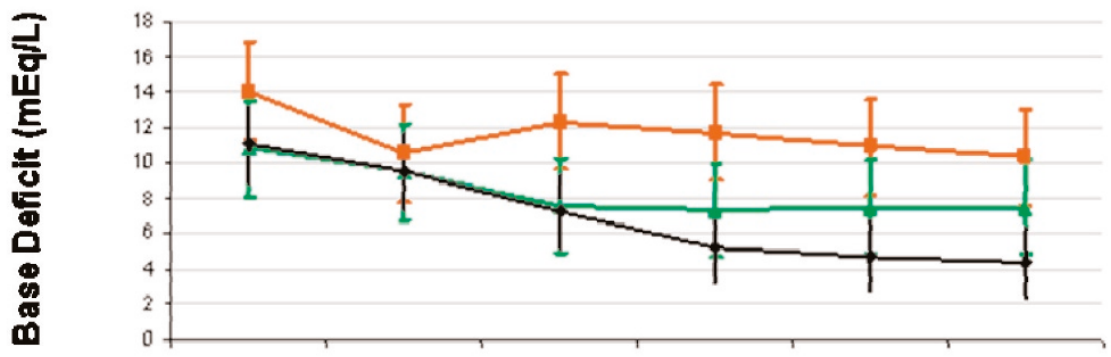

Hour $<0.0001$ Group 0.0101 Hr*Grp 0.0040

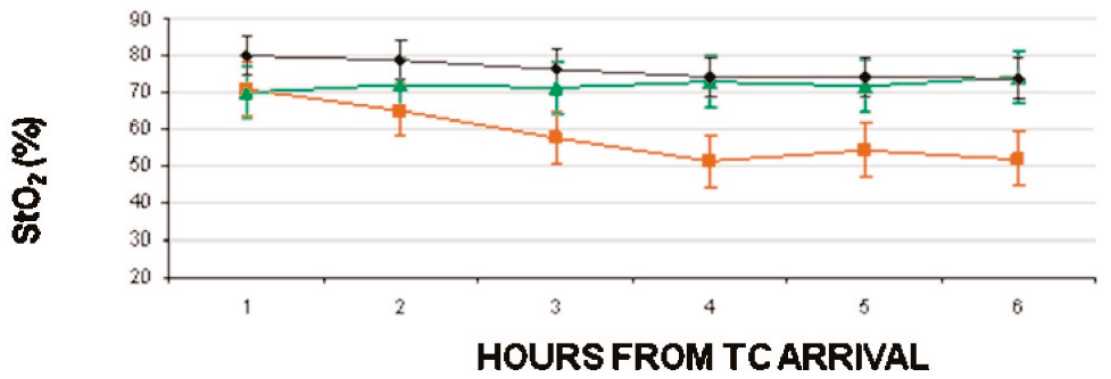

Hour 0.0053 Group $<0.0001$ $\mathrm{Hr}^{*} \mathrm{Grp} 0.0089$

Shock indices over the first 6 hours of hospitalization. G1, massive transfusion (MT) and dies in $\leq 24$ hours; G2, MT and dies in $>24$ hours and/or multiple organ failure (MOF); G3, MT and survived without MOF. $P$-values reported for the group and hour from trauma center (TC) arrival factor and the interaction term. SBP, systolic blood pressure; $\mathrm{StO}_{2}$, tissue hemoglobin oxygen saturation. Reproduced with permission from [26].

\section{Conclusion}

In our ongoing experience, $\mathrm{NIRS}$ or $\mathrm{StO}_{2}$ monitoring offers a continuous non-invasive monitor of hypoperfusion. In early clinical testing during active shock resuscitation, changes in skeletal muscle $\mathrm{StO}_{2}$ correlated well with changes in $\mathrm{DO}_{2}$, base deficit and lactate levels. This clinical experience in active shock resuscitation has likewise been verified in laboratory studies. In a prospective clinical trial of $\mathrm{StO}_{2}$ monitoring obtained within the first hour after ED admission of major torso trauma patients who were presumed to be bleeding, the $\mathrm{StO}_{2}$ value predicted death and MOF as well as or perhaps better than base deficit and lactate levels [15]. 
Additionally, in the setting of MT we observed that a drop in $\mathrm{StO}_{2}$ portends early death from exsanguination $[25,26]$ and may be helpful in making critical decisions.

\section{Competing interests}

FAM is a member of the Hutchinson Technology Inc. Trauma and Critical Care Advisory Board. RJS declares that they have no competing interests.

\section{Acknowledgement}

This article is part of Critical Care Volume 13 Supplement 5: Tissue oxygenation $\left(\mathrm{StO}_{2}\right)$ in healthy volunteers and critically-ill patients. The full contents of the supplement are available online at http://ccforum. $\mathrm{com} /$ supplements/13/S5. Publication of the supplement has been supported with funding from Hutchinson Technology Inc.

\section{References}

1. Shoemaker WC, Appel P, Bland R: Use of physiologic monitoring to predict outcome and to assist in clinical decisions in critically ill postoperative patients. Am J Surg 1983, 146:43-50.

2. Shoemaker WC, Appel PL, Kram HB, Waxman K, Lee TS: Prospective trial of supranormal values survivors as therapeutic goals in high risk surgical patients. Chest 1988, 94: 1176-1183.

3. Shoemaker WC: Invasive and noninvasive hemodynamic monitoring of high-risk patients to improve outcome. Semin Anesth Periop Med Pain 1999, 18:63-70.

4. Nelson L: Continous venous oximetry in surgical patients. Ann Surg 1986, 203:329-333.

5. Moore FA, Haenal J, Moore E, Whitehall T: Incommensurate oxygen consumption in response to maximal oxygen availability predicts postinjury multiple organ failure. J Trauma 1992, 33:58-65.

6. Kern JW, Shoemaker WC: Meta-analysis of hemodynamic optimization in high risk patients. Crit Care Med 2002, 30:16861692.

7. Moore FA: Evidence-based medical information technology: the next generation. J Trauma 2007, 63:1195-1205.

8. McKinley BA, Marvin R, Coconour CS, Moore FA: Tissue hemoglobin $\mathrm{O}_{2}$ saturation during resuscitation of traumatic shock monitored by using near infrared spectrometry. J Trauma 2000, 48:637-642.

9. Taylor JH, Mulier KE, Myers DE, Beilman GJ: Use of near infrared spectroscopy in early determination of irreversible hemorrhagic shock. J Trauma 2005, 58:1119-1125.

10. Crookes BA, Cohn SM, Burton EA, Nelson J, Proctor KG: Noninvasive muscle oxygenation to guide fluid resuscitation after shock. Surgery 2004, 135:662-670.

11. Davis J, Shckford SR, Mackersie RC, Hoyt DB: Base deficit as a guide to volume resuscitation. J Trauma 1988, 28:1464-1467.

12. Davis JW, Parks SN, Kaups KL, Gladen HE, O'Donnell-Nicol S: Admission base deficit predicts transfusion requirements and risk of complications. J Trauma 1996, 41:769-774.

13. Sauaia A, Moore FA, Moore EE, Haenal JB, Read RA, Lezotte DC: Early predictors of postinjury multiple organ failure. Arch Surg 1994, 129:39-45.

14. Sauaia A, Moore FA, Moore EE, Norris JM, Lezotte DC: Multiple organ failure can be predicted as early as $\mathbf{1 2}$ hours after injury. J Trauma 1998, 45:291-301.

15. Cohn SM, Nathens AB, Moore FA, Rhee P, Puyana JC, Moore EE, Beilman GJ, and the $\mathrm{StO}_{2}$ in Trauma Patients Trail Investigators: Tissue oxygen saturation predicts the development of organ dysfunction during traumatic shock resuscitation. J Trauma 2007, 62:44-55.

16. Moore FA: Tissue oxygen saturation predicts the development of organ failure during traumatic shock resuscitation. In International Proceedings of the 7th World Congress on Trauma, Shock, Inflammation and Sepsis; 13-17 March 2007; Munich, Germany. Edited by E Faist. Bologna, Italy: Medimond; 2007:111-114.

17. Zsolt B, McKinley BA, Coconour CS, Kozar RA, Holcomb J, Ware $D$, Moore FA: Secondary abdominal compartment syndrome is an elusive early complication of shock resuscitation. $\mathrm{Am} \mathrm{J}$ Surg 2002, 184:538-543.
18. Zsolt B, McKinley BA, Holcomb J, Miller CC, Coconour CS, Kozar RA, Valdivia A, Ware DN, Moore FA: Both primary and secondary abdominal compartment syndrome can be predicted early and harbingers of multiple organ failure. J Trauma 2003, 54:848-861.

19. Zsolt B, McKinley BA, Coconour CS, Kozar RA, Cox C, Moore FA: Patients with impending abdominal compartment syndrome do not respond to early volume loading. Am J Surg 2003, 186: 602-607.

20. Zsolt B, McKinley BA, Coconour CS, Kozar RA, Valdivia A, Sailors $M$, Moore FA: Supranormal trauma resuscitation causes more cases of abdominal compartment syndrome. Arch Surg 2003, 138:637-642.

21. Zsolt B, McKinley BA, Cox C, Allen SJ, Coconour CS, Kozar RA, Moore EE, Miller CC, Weisbrodt NW, Moore FA: Abdominal compartment syndrome: the cause of effect of postinjury multiple organ failure. Shock 2003, 20:483-492.

22. Gonzalez EA, Moore FA, Holcomb JB, Miller CC, Kozar RA, Todd SR, Cocanour CS, Balldin BC, McKinley BA: Fresh frozen plasma should be given earlier to patients requiring massive transfusion. J Trauma 2007, 62:112-119.

23. Holcomb JB, Hoots WK, Moore FA: Treatment of an acquired coagulopathy with recombinant activated factor VII in a damage-control patient. Mil Med 2005, 170:287-290.

24. Holcomb JB, Jenkins $D$, Rhee $P$, Johannigman J, Mahoney $P$ Mehta S, Cox ED, Gehrke MJ, Beilman GJ, Schreiber M, Flaherty SF, Grathwohl KW, Spinella PC, Perkins JG, Beekley AC, McMullin NR, Park MS, Gonzalez EA, Wade CE, Dubick MA Schwab CW, Moore FA, Champion HR, Hoyt DB, Hess JR: Damage control resuscitation: directly addressing the early coagulopathy of trauma. J Trauma 2007, 62:307-310.

25. Moore FA, Nelson T, McKinley BA, Moore EE, Nathen AB, Rhee P, Puyana JC, Beilman GJ, Cohn SM, and the $\mathrm{StO}_{2}$ Study Group: Massive transfusion in trauma patients: tissue hemoglobin oxygen saturation predicts bad outcomes. J Trauma 2008, 64: 1010-1023.

26. Moore FA, Nelson T, McKinley BA, Moore EE, Nathen AB, Rhee $\mathrm{P}$, Puyana JC, Beilman GJ, Cohn SM, and the $\mathrm{StO}_{2}$ Study Group: Is there a role for aggressive use of fresh frozen plasma in massive transfusion of civilian trauma patients. Am J Surg 2008, 196:948-960. 